\title{
Discontinuous subgrid formulations for transport problems
}

\author{
Natalia C.B. Arruda ${ }^{a}$, Regina C. Almeida ${ }^{\text {b, } * \text {, Eduardo G. Dutra do Carmo }}{ }^{\mathrm{c}}$ \\ a The Computational Modeling Program, Laboratório Nacional de Computação Científica - LNCC/MCT, Av. Getúlio Vargas, 333 Petrópolis, RJ 25651-075, Brazil \\ ${ }^{\mathrm{b}}$ Department of Computational Mechanics, Laboratório Nacional de Computação Científica - LNCC/MCT, Av. Getúlio Vargas, 333 Petrópolis, RJ 25651-075, Brazil \\ ${ }^{\mathrm{c}}$ Department of Nuclear Engineering, COPPE/UFRJ - Universidade Federal do Rio de Janeiro, P.B. 68503, Rio de Janeiro, RJ, Brazil
}

\section{A R T I C L E I N F O}

\section{Article history:}

Received 29 September 2009

Received in revised form 12 April 2010

Accepted 24 June 2010

Available online 1 July 2010

\section{Keywords:}

Discontinuous Galerkin

Two-scale finite element model

Advection-diffusion-reaction equations

\begin{abstract}
A B S T R A C T
In this paper we develop two discontinuous Galerkin formulations within the framework of the two-scale subgrid method for solving advection-diffusion-reaction equations. We reformulate, using broken spaces, the nonlinear subgrid scale (NSGS) finite element model in which a nonlinear eddy viscosity term is introduced only to the subgrid scales of a finite element mesh. Here, two new subgrid formulations are built by introducing subgrid stabilized terms either at the element level or on the edges by means of the residual of the approximated resolved scale solution inside each element and the jump of the subgrid solution across interelement edges. The amount of subgrid viscosity is scaled by the resolved scale solution at the element level, yielding a self adaptive method so that no additional stabilization parameter is required. Numerical experiments are conducted in order to demonstrate the behavior of the proposed methodology in comparison with some discontinuous Galerkin methods.
\end{abstract}

(c) 2010 Elsevier B.V. All rights reserved.

\section{Introduction}

Advection-diffusion-reaction equations have important applications in modeling many natural phenomena in engineering, environmental protection, petroleum industry, hydrology, etc. They are also suitable models to study numerical methods for flow problems, which can be later applied to more complex problems since they present similar computational challenges. These mainly appear due to the domination of the advection and/or the reaction phenomena, parameters discontinuity or inhomogeneous boundary conditions. In these cases, the smallest scales of the computational grid may not be fine enough for the viscous dissipation to be effective. The presence of considerable information contained in small scales (smaller than the grid scale), whose effects are not captured, gives rise to spurious modes in the desired solution represented by the approximation space. Actually, for many flows in nature, it is impossible to capture all the scales in a numerical simulation since the scale separation may span several orders of magnitude [1].

One approach to incorporate the effects of the small scales on the resolved grid scale uses multiscale methods. The variational multiscale framework consists in decomposing the variable of interest into a resolved coarse scale and an unresolved subgrid

\footnotetext{
* Corresponding author. Tel.: +55 24 22336155; fax: +55 2422336165 .

E-mail addresses: nataliac@lncc.br (N.C.B. Arruda), rcca@Incc.br (R.C. Almeida), egdcarmo@hotmail.com (E.G. Dutra do Carmo).
}

scale. As point out in [2], this framework allowed to reformulate the well known stabilized formulations as techniques which incorporate missing unresolved fine-scale variability into the coarse-scale solution. Examples of these methods are: residual free bubbles (RFB) [3], variational multiscale (VMS) [4,5] and subgrid stabilization (SGS) [6,7]. Like stabilized methods, many multiscale methods also depend on some user-defined stabilization coefficient, which can be both mesh and problem dependent. This is the case of the SGS method, which can also be seen as an eddy viscosity model that is introduced on only the subgrid scales. Based on that idea, a nonlinear subgrid eddy viscosity model, the nonlinear subgrid stabilization (NSGS), was developed in [8], in which the amount of the eddy viscosity is locally and adaptively controlled by the resolved scale solution. The NSGS keeps the simplicity of a subgrid viscosity model, does not depend on any tunable parameter and precludes local and nonlocal oscillations in many singular perturbed problems.

On the other hand, discontinuous Galerkin (DG) methods have been widely used in the science and engineering community in order to solve advection dominated problems. DG methods handle rough coefficient problems and capture discontinuities in the solution very well by the nature of discontinuous function approximation space. They have less numerical diffusion than most conventional algorithms. The DG methods are efficient in handling inhomogeneous boundary conditions, in performing $h-p$ adaptivity and, because of the simple communication pattern between elements, they are easily paralelizable. They can be advantageously used in a broad range of applications, including problems involving domain decomposition $[9,10]$. However, DG solutions may suffer 
from non-physical oscillations in the neighborhood of discontinuities that exist, for example, in problems governed by hyperbolic conservation laws [11]. Thus, some additional stabilization may be necessary, depending on the problem. Some approaches to address this issue are based on slope limiters [12-14], PetrovGalerkin stabilizations [15,16], bubble stabilization [17,18], interior penalty-type stabilizations $[16,19]$ and subgrid stabilization [1]. The subgrid method introduced in the latter work is a combination of a DG method with a linear eddy viscosity model, which is also controlled by a user-defined mesh dependent coefficient $v_{T}$. That method was analyzed when applied to the Navier-Stokes equations, yielding optimal convergence rates with respect to the mesh size which depend on the Reynolds number in a reasonable fashion [1]. However, as discontinuous approximation spaces are used for both the resolved and unresolved variables, the method involves a huge number of degrees of freedom. Another drawback is the necessity of selecting a stabilization coefficient, which is a tricky task for actual problems. On the other hand, the use of edge stabilization has its origins in the seventies, when continuous interior penalty finite element methods were introduced by Babuška and Zlámal [20], for the biharmonic operator, and by Douglas and Dupont [21], for second-order elliptic and parabolic problems. The key idea is to relax the $C^{1}$ continuity by means of a penalty on the jump in the normal derivative across the interior edges of elements. More recently, this technique was improved for continuous and discontinuous finite element methods [22,23]. In [19], the main stabilization is provided by an interior penalty-type term, which is proved to be sufficient not only for discontinuous Galerkin method but also for conforming piecewise linear finite element approximations.

The combination of DG methods and subgrid stabilization seems attractive since it allows for capturing of advection effects and taking into account the dispersive effects of small scales on the large scales correctly. The approximate solution can be strongly improved inserting the subgrid viscosity only where it is necessary, using a procedure similar to that introduced in the NSGS method. Thus, in this work we present two new formulations which reformulates the NSGS method using broken spaces. The new methods improve stability while being more economic than classical DG methods since we allow discontinuities only between the element edges of the coarsest mesh, reducing the number of degreesof-freedom. Both approaches start with a two-level decomposition of the domain: the coarsest is a triangular partition $\mathcal{T}_{H}$ of the domain and the more refined one is obtained by subdividing each macro (coarsest) element into four micro triangles by connecting the macro midpoints edges $\left(\mathcal{T}_{h}\right)$. The broken spaces are defined on the coarsest partition. The approximation space $X_{h}$, defined on $\mathcal{T}_{h}$, is split into resolved scales $\left(X_{H}\right)$ and subgrid scales $\left(X_{H}^{h}\right)$ spaces so that it is continuous inside each macro element. The first formulation is built by considering a subgrid eddy viscosity model, à la NSGS, and an additional subgrid edge stabilization term. They both are nonlinear and depend on the resolved scale solution, yielding a self adaptive method. The second formulation is inspired by [19] and introduces a subgrid edge discontinuous model that also depends on the resolution of the resolved scale solution and so adapts the edge stabilization according to the local problem. The two formulations have similar stability and convergence properties and are consistent.

The outline of this paper is as follows. Section 2 briefly addresses the model problem and notation. Section 3 presents the first new discontinuous formulation based on a nonlinear subgrid eddy model. The edge subgrid stabilization is introduced in Section 4. Numerical experiments are conducted in Section 5 to show the behavior of the proposed methodologies for a variety of transport problems. Section 6 concludes this paper.

\section{Preliminaries}

We consider the advection-diffusion-reaction equation as follows:

$-\varepsilon \Delta u+\boldsymbol{\beta} \cdot \nabla u+\sigma u=f, \quad$ in $\Omega ;$

$u=g$ on $\Gamma$,

where $\Omega \subset \mathbb{R}^{d}(d=2)$ is an open bounded domain with a Lipschitz boundary $\Gamma, \boldsymbol{\beta}$ is the divergence free velocity field, $\sigma$ is the reaction coefficient, $0<\varepsilon \ll 1$ is the (constant) diffusion coefficient and $f$ is the source term. For simplicity we have considered here Dirichlet boundary conditions. All the results of this paper can be extended to deal with general boundary conditions.

We define the inflow and outflow parts of $\Gamma$, respectively, in the usual fashion:

$\Gamma_{-}=\{x \in \Gamma: \boldsymbol{\beta}(x) \cdot \mathbf{n}(x)<0\} ;$

$\Gamma_{+}=\{x \in \Gamma: \boldsymbol{\beta}(x) \cdot \mathbf{n}(x)>0\}$,

where $\mathbf{n}(x)$ denotes the unit outward normal vector to $\Gamma$ at $x \in \Gamma$.

In order to derive a DG formulation for (1), we have to introduce some notation. The subgrid stabilization considered here is based on a two-level discretization so that two nested grids must be built. Let consider a coarsest regular triangulation $\mathcal{T}_{H}$ of the domain $\Omega$ into triangles $T_{H}$, where $H$ stands for the diameter of $T_{H}$ in $\mathcal{T}_{H}$. For each triangle $T_{H} \in \mathcal{T}_{H}$, four triangles are created by connecting the midpoint of the edges. We set $h=H / 2$ and denote by $\mathcal{T}_{h}=\left\{T_{h}\right\}$ the resulting finer triangulation. Let $\mathcal{E}_{h}$ be the set of edges of $\mathcal{T}_{h}$. Let $e_{M}=\left\{e_{1}, e_{2}\right\}, e_{j} \in \mathcal{E}_{h}, j=1,2$, be an edge of a macro triangle $T_{H} \in \mathcal{T}_{H}$. Fig. 1 shows the particular case when $e_{M}$ is an interior edge shared by the macro triangles $T_{1}, T_{2} \in \mathcal{T}_{H}$ and $e_{j} \in e_{M}$ is shared by triangles $T_{1, j}, T_{2, j} \in \mathcal{T}_{h}$. The set of all edges of $\mathcal{T}_{H}$ is then defined by $\mathcal{E}_{H}=\cup_{\mathcal{T}_{H}} e_{M}$. We also define $\mathcal{E}=\mathcal{E}_{h} \cap \mathcal{E}_{H}=\mathcal{E}^{0} \cup \mathcal{E}^{\Gamma}$, where $\mathcal{E}^{0}$ and $\mathcal{E}^{\Gamma}$ are the sets of internal edges and of edges on the boundary $\Gamma$, respectively. The generic edge $e$ that is in an inflow part of the domain belongs to the set $\mathcal{E}^{0-}=\left\{e \in \mathcal{E}^{0}: \boldsymbol{\beta}(x) \cdot \mathbf{n}(x)<0\right.$, $\forall x \in e\}$, if it is an interior edge, or to $\mathcal{E}_{h}^{\Gamma_{-}}=\mathcal{E}^{\Gamma} \cap \Gamma_{-}$, if it lies on the boundary. Moreover, let $\mathbf{n}^{1}$ and $\mathbf{n}^{2}$ be the unit normal vectors on $e_{j}$ pointing outward to $T_{1 j}$ and $T_{1 j}$, respectively. For a scalar function $\varphi$, piecewise smooth on $\mathcal{T}_{h}$, with $\varphi^{i}:=\left.\varphi\right|_{T_{i, j}}$ for each edge $e_{j}, j=1,2$, we define:

$\{\varphi\}=\frac{1}{2}\left(\varphi^{1}+\varphi^{2}\right), \quad \llbracket \varphi \rrbracket=\varphi^{1} \mathbf{n}^{1}+\varphi^{2} \mathbf{n}^{2} \quad$ on $e_{j} \in \mathcal{E}^{0}$,

the average and the jump operators of a function $\varphi$ on $e_{1}, e_{2} \in e_{M} \subset$ $\mathcal{E}_{H}$, respectively. For $e_{j} \in \mathcal{E}^{\Gamma}$, the jump and average of $\varphi$ coincide with its trace on $e_{j}$. For a vector-valued function $\tau$, piecewise smooth on $\mathcal{T}_{h}$, with $\tau^{i}:=\left.\tau\right|_{T_{i, j}}, j=1,2$, we also define:

$\{\tau\}=\frac{1}{2}\left(\tau^{1}+\tau^{2}\right), \quad \llbracket \tau \rrbracket=\tau^{1} \cdot \mathbf{n}^{1}+\tau^{2} \cdot \mathbf{n}^{2} \quad$ on $e_{j} \in \mathcal{E}^{0}$

A two-level finite element approximation is defined by introducing the following two broken spaces on the partition $\mathcal{T}_{H}$ :
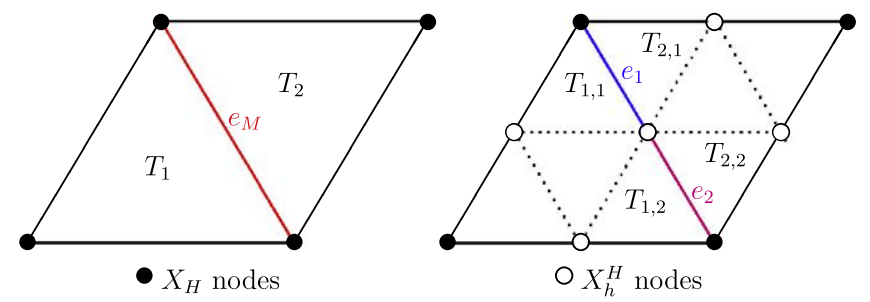

Fig. 1. Schematic representation of the two-level $\mathbb{P}_{1}$ setting. 


$$
\begin{aligned}
X_{H}= & \left\{u_{H} \in L^{2}(\Omega)\left|u_{H}\right|_{T_{H}} \in H^{1}\left(T_{H}\right) \cap \mathbb{P}_{k}\left(T_{H}\right), \quad \forall T_{H} \in \mathcal{T}_{H}\right\} ; \\
X_{h}= & \left\{u_{h} \in L^{2}(\Omega)\left|u_{h}\right|_{T_{H}} \in H^{1}\left(T_{H}\right),\left.\quad u_{h}\right|_{T_{h}} \in \mathbb{P}_{k}\left(T_{h}\right),\right. \\
& \left.\forall T_{h} \in \mathcal{T}_{h}, \quad \forall T_{H} \in \mathcal{T}_{H}\right\},
\end{aligned}
$$

where $\mathbb{P}_{k}$ stands for the set of interpolation polynomials of degree less or equal to $k$. In this paper we set $k=1$ and we denote the finite element approximation defined by the couple $\left(X_{H}, X_{h}\right)$ by two-level $\mathbb{P}_{1}$. Higher order polynomials may be used as well.

We also introduce an additional discrete space $X_{h}^{H} \subset X_{h}$, such that the following decomposition holds:

$X_{h}=X_{H} \oplus X_{h}^{H}$,

where $X_{H}$ is the resolved (coarse) scale space whereas $X_{h}^{H}$ is the subgrid (refined) scale space. Given $u_{h} \in X_{h}$ and $u_{H} \in X_{H}$ such that $u_{h}$ and $u_{H}$ coincide in the coarse-scale nodes, we define $u_{h}^{H} \in X_{h}^{H}$ and the space decomposition (8) implies that $u_{h}^{H}=u_{h}-u_{H}$. More precisely, let $P_{H}: X_{h} \rightarrow X_{H}$ be the projection of $X_{h}$ onto $X_{H}$ that is parallel to $X_{h}^{H}$. For all $v_{h} \in X_{h}$ we set $v_{H}=P_{H} v_{h}$ and $v_{h}^{H}=\left(I-P_{H}\right) v_{h}$. The definition of $P_{H}$ for the two-level $\mathbb{P}_{1}$ setting defined by the couple $\left(X_{H}, X_{h}\right)$ is given in [24, p. 242].

One may notice that the space $X_{h}$ is required to be continuous inside of each $T_{H}$, although it is discontinuous along $e_{M} \subset \mathcal{E}_{H}$. As a two-level piecewise linear finite element approximation is adopted, the subgrid scale solution may be nonzero across the $\mathcal{T}_{H}$ meshlines. This property will be used to build the new two nonlinear discontinuous subgrid formulations.

\section{Nonlinear subgrid discontinuous formulation - NSDG}

The new subgrid discontinuous stabilization proposed here extends the nonlinear subgrid stabilization, recently proposed in [8], to discontinuous formulations. The NSGS method introduces a nonlinear artificial viscosity controlled by a minimization process of the kinetic energy associated to the unresolved scales. The main idea in [8] is to decompose the velocity field into the resolved (coarse) and unresolved (subgrid) scales, with respect to the grid scales, as $\boldsymbol{\beta}=\boldsymbol{\beta}_{H}+\boldsymbol{\beta}_{h}^{H}$. The subgrid velocity field $\boldsymbol{\beta}_{h}^{H}$ is determined by requiring the minimum of the associated kinetic energy for which the residual of the resolved scale solution (with $\boldsymbol{\beta}_{H}$ ) vanishes. The solution of this minimization problem yields a subgrid velocity field that is effectively a projection of the coarse-scale residual along the gradient of the resolved scale solution when $\nabla u_{H} \neq 0$ in the form:

$\boldsymbol{\beta}_{h}^{H}=\frac{R\left(u_{H}\right)}{\left|\nabla u_{H}\right|^{2}} \nabla u_{H}$,

where $R\left(u_{H}\right)=-\varepsilon \Delta u_{H}+\boldsymbol{\beta} \cdot \nabla u_{H}+\sigma u_{H}-f$. When $\nabla u_{H}=0$, the subgrid velocity vanishes. The subgrid velocity (9) is used to define an eddy viscosity term that is introduced only on the subgrid scales.

A straightforward extension of the NSGS to the discontinuous Galerkin framework would read: find $u_{h} \in X_{h}$ such that:

$$
\begin{aligned}
\widehat{B}_{\mathrm{DG}}\left(u_{h}, v_{h}\right) & =B\left(u_{h}, v_{h}\right)+B_{E}\left(u_{h}, v_{h}\right)+D_{\mathrm{NSGS}}\left(u_{H}, u_{h}, v_{h}\right) \\
& =L\left(v_{h}\right)+L_{E}\left(v_{h}\right), \quad \forall v_{h} \in X_{h},
\end{aligned}
$$

where

$$
\begin{aligned}
B\left(u_{h}, v_{h}\right)= & \sum_{T \in \mathcal{T}_{h}} \int_{T}\left[\varepsilon \nabla u_{h} \cdot \nabla v_{h}+\left(\boldsymbol{\beta} \cdot \nabla u_{h}\right) v_{h}+\sigma u_{h} v_{h}\right] d \Omega ; \\
B_{E}\left(u_{h}, v_{h}\right)= & \sum_{e \in \mathcal{E}^{0}} \int_{e}\left[\llbracket u_{h} \rrbracket \cdot\left\{\varepsilon \nabla v_{h}\right\}-\left\{\varepsilon \nabla u_{h}\right\} \cdot \llbracket v_{h} \rrbracket+\eta_{1} \llbracket u_{h} \rrbracket \cdot \llbracket v_{h} \rrbracket\right] d S \\
& -\sum_{e \in \mathcal{E}^{0-}} \int_{e} \boldsymbol{\beta} \cdot \llbracket u_{h} \rrbracket v_{h} d S-\sum_{e \in \mathcal{E}^{\Gamma-}} \int_{e}(\boldsymbol{\beta} \cdot \mathbf{n}) u_{h} v_{h} d S \\
& +\sum_{e \in \mathcal{E}^{\Gamma}} \int_{e}\left[u_{h}\left(\varepsilon \nabla v_{h} \cdot \mathbf{n}\right)-\left(\varepsilon \nabla u_{h} \cdot \mathbf{n}\right) v_{h}+\eta_{1} u_{h} v_{h}\right] d S ;
\end{aligned}
$$

$$
\begin{aligned}
& L\left(v_{h}\right)=\sum_{T \in \mathcal{T}_{h}} \int_{T} f v_{h} d \Omega \\
& L_{E}\left(v_{h}\right)=\sum_{e \in \mathcal{E}^{T}} \int_{e}\left[g\left(\varepsilon \nabla v_{h} \cdot \mathbf{n}\right)+\eta_{1} g v_{h}\right] d S-\sum_{e \in \mathcal{E}^{T^{-}}} \int_{e}(\boldsymbol{\beta} \cdot \mathbf{n}) g v_{h} d S
\end{aligned}
$$

$$
D_{\mathrm{NSGS}}\left(u_{H}, u_{h}, v_{h}\right)= \begin{cases}\sum_{T \in \mathcal{T}_{h}} \int_{T} \xi_{h}^{H} \nabla u_{h}^{H} \cdot \nabla v_{h}^{H} d \Omega, & \text { if } \nabla u_{H} \neq 0 \\ 0, & \text { if } \nabla u_{H}=0\end{cases}
$$

and $\eta_{1}=4 \varepsilon / s, \quad s=\min \left\{\operatorname{meas}\left(T_{1 j}\right)^{1 / d}, \operatorname{meas}\left(T_{2 j}\right)^{1 / d}\right\}, j \in\{1,2\}$, where $T_{1, j}, T_{2, j} \in \mathcal{T}_{h}$ are the triangles sharing an interior edge $e$. The amount of subgrid viscosity in (15) is defined as

$\xi_{h}^{H}=\frac{1}{2} \mu(h)\left|\boldsymbol{\beta}_{h}^{H}\right|$,

where $\mu(h)$ characterizes the subgrid length scale and is usually assumed to be equal to meas $\left(T_{h}\right)^{1 / d}$. The bilinear forms $B(\cdot, \cdot)$ and $B_{E}(\cdot, \cdot)$, and the functionals $L(\cdot)$ and $L_{E}(\cdot)$ are associated to the discontinuous Galerkin formulation developed in [15]. However, since the fine-scale solution $u_{h}$ is required to be continuous inside $T_{H} \in \mathcal{T}_{H}$, the formulation (10) (without the nonlinear operator (15)) is different from that in [15] and yields fewer number of degrees of freedom. From now on, this linear discontinuous formulation is denoted by $\mathrm{DG}_{s}$. One may also notice that the subgrid stabilization term (15) vanishes when $\nabla u_{H}=0$, following the improvement of the continuous NSGS method presented in [25].

The formulation (10) does not take into account the fact that the subgrid solution may not vanish on the edges of $T_{H}$. Actually, it seems natural to require $\llbracket u_{h}^{H} \rrbracket=0$ almost everywhere on $\mathcal{E}^{0}$. This residual equation of the subgrid jumps on $\mathcal{E}^{0}$ may be weakly satisfied by supplementing (10) with a penalty term of the subgrid jumps, enhancing stability. Hence, the new discontinuous nonlinear subgrid scale formulation (NSDG) proposed here is given by: find $u_{h} \in X_{h}$ such that:

$$
\begin{aligned}
B_{\mathrm{NSDG}}\left(u_{h}, v_{h}\right) & =B\left(u_{h}, v_{h}\right)+B_{E}\left(u_{h}, v_{h}\right)+D_{\mathrm{NSDG}}\left(u_{H}, u_{h}, v_{h}\right) \\
& =L\left(v_{h}\right)+L_{E}\left(v_{h}\right), \quad \forall v_{h} \in X_{h},
\end{aligned}
$$

where

$$
\begin{aligned}
D_{\mathrm{NSDG}}\left(u_{H}, u_{h}, v_{h}\right)= & \sum_{T \in \mathcal{T}_{h}} \int_{T} \xi_{h}^{H} \nabla u_{h}^{H} \cdot \nabla v_{h}^{H} d \Omega+\sum_{e \in \mathcal{E}^{0}} \int_{e} \eta_{2} \llbracket u_{h}^{H} \rrbracket \\
& \cdot \llbracket v_{h}^{H} \rrbracket d S, \quad \text { if } \nabla u_{H} \neq 0 ;
\end{aligned}
$$

and $D_{\mathrm{NSDG}}\left(u_{H}, u_{h}, v_{h}\right)=0$ if $\nabla u_{H}=0$, where $\eta_{2}=4 \xi_{h}^{H} / s$. The first term of (18) acts inside each element of $\mathcal{T}_{H}$ ( or $\mathcal{T}_{h}$ ), introducing the necessary amount of artificial viscosity to dissipate the kinetic energy associated to the unresolved scales. The second term, weighted by $\eta_{2}$, introduces a penalty of the unresolved scale jumps. Since $\eta_{2}$ depends on $\xi_{h}^{H}$, which depends on the residual of the resolved scale solution, this penalty term is also self adaptive.

The operator $B_{\mathrm{NSDG}}(\cdot, \cdot)$ is defined only on the discrete space $X_{h} \times X_{h}$ and the exact solution $u$ has a priori no decomposition in $X_{H} \oplus X_{h}^{H}$. However, by definition, both the exact solution and the resolved scale solution $u_{H}$ have no subgrid scales (see [26, p. 256]). Thus, it follows that the operator $D_{\mathrm{NSDG}}\left(u_{H}, u_{h}, v_{h}\right)$ vanishes for every strong solution of (1), yielding the consistency property.

The nonlinear formulation (17) is solved using a quite simple iterative procedure. For each iterative step, the subgrid artificial viscosity is delayed one iteration and the $\mathrm{DG}_{s}$ solution for the resolved scales is adopted as the initial guess. The convergence is checked for all the resolved scale degrees of freedom, under a prescribed tolerance. 


\section{Edge subgrid discontinuous formulation - ESDG}

As mentioned before, the edge stabilization technique for finite element methods for second-order elliptic and parabolic problems was originally proposed by Douglas and Dupont [21], by means of a least square stabilization of the gradient jumps across element boundaries. This procedure was recalled by Burman and Hansbo [22], in which the stability in the hyperbolic limit and optimal error estimates are proved. In [19] a unified analysis of this technique for conforming and nonconforming finite element methods was developed.

The edge subgrid stabilization proposed here is based on two higher-order interior penalty terms. The main idea is to control the part of the streamline derivative that cannot be represented by the approximation space. Thus, the proposed edge subgrid discontinuous formulation (ESDG) is given by: find $u_{h} \in X_{h}$ such that:

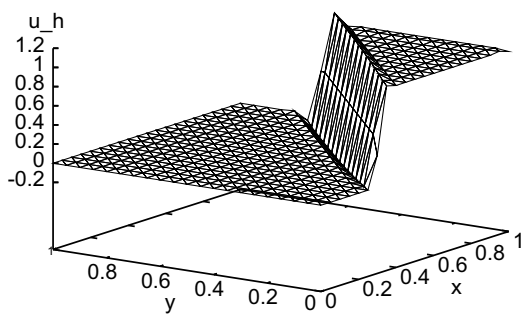

(a) DG solution.

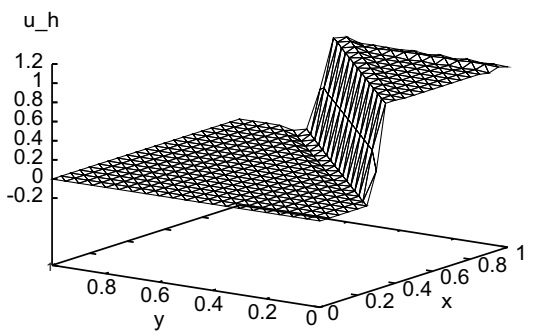

(c) NSDG solution $\left(u_{h}\right)$.

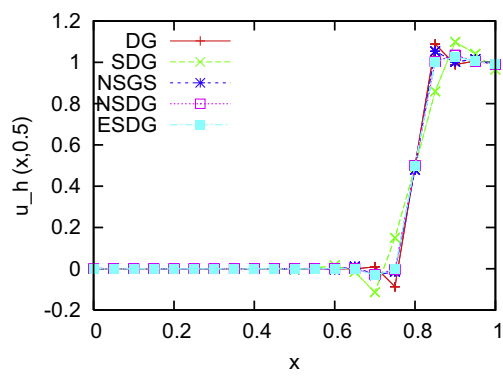

(e) Section at $y=0.5$.

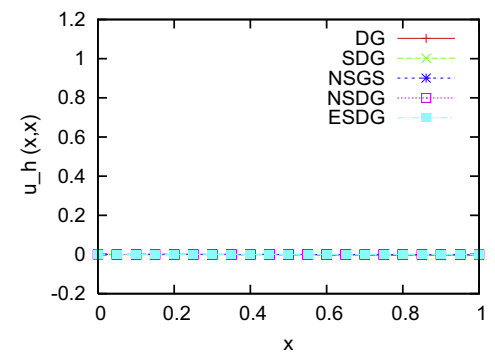

(g) Section at $y=x$.

$$
\begin{aligned}
B_{\mathrm{ESDG}}\left(u_{h}, v_{h}\right) & =B\left(u_{h}, v_{h}\right)+B_{E}\left(u_{h}, v_{h}\right)+D_{\mathrm{ESDG}}\left(u_{H}, u_{h}, v_{h}\right) \\
& =L\left(v_{h}\right)+L_{E}\left(v_{h}\right), \quad \forall v_{h} \in X_{h},
\end{aligned}
$$

where

$$
\begin{aligned}
D_{\mathrm{ESDG}}\left(u_{H}, u_{h}, v_{h}\right)= & \sum_{e \in \mathcal{E}^{0}} \frac{\gamma_{1} h^{2}}{F} \int_{e} \llbracket \beta_{h}^{H} \cdot \nabla u_{h}^{H} \rrbracket \cdot \llbracket \beta_{h}^{H} \cdot \nabla v_{h}^{H} \rrbracket d S \\
& +\sum_{e \in \mathcal{E}^{0}} \frac{1}{2} \frac{\gamma_{2} h^{2}}{F} \int_{e}\left[\beta^{T_{1} T_{2}, H} \cdot\left\{\nabla u_{h}^{H}\right\}\right]\left[\beta^{T_{1} T_{2}, H} \cdot\left\{\nabla v_{h}^{H}\right\}\right] d S,
\end{aligned}
$$

with $\gamma_{1}, \gamma_{2}>0, h=\min \left\{h_{e, T_{1, j}}, h_{e, T_{2, j}}\right\}$, where $h_{e, T_{i, j}}=h_{e_{j}, T_{i, j}}$ is the length of the edge shared by $T_{1, j}$ and $T_{2, j}, j \in\{1,2\}$, and $\beta^{T_{1} T_{2}, H}$ is given by

$\beta^{T_{1} T_{2}, H}=\frac{\left|R\left(u_{H}\right)_{\mid T_{1}}+R\left(u_{H}\right)_{\mid T_{2}}\right|}{\left|\nabla u_{H_{\mid T_{1}}}+\nabla u_{H_{\mid T_{2}}}\right|^{2}}\left(\nabla u_{H_{\mid T_{1}}}+\nabla u_{H_{\mid T_{2}}}\right)$.

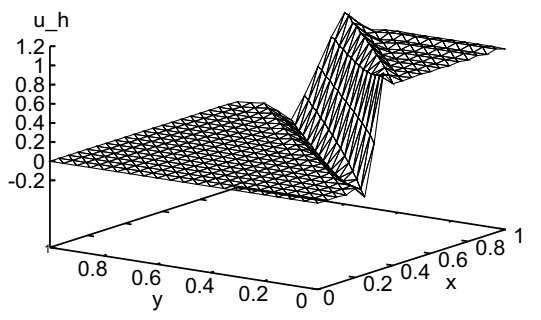

(b) SDG solution $\left(u_{h}\right)$.

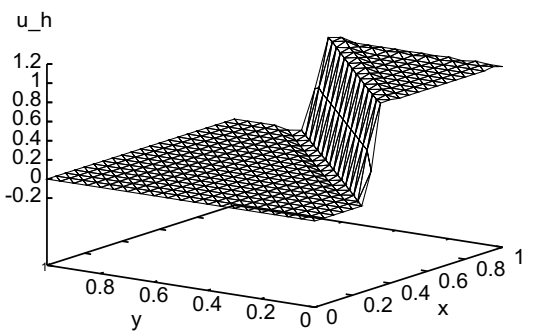

(d) ESDG solution $\left(u_{h}\right)$.

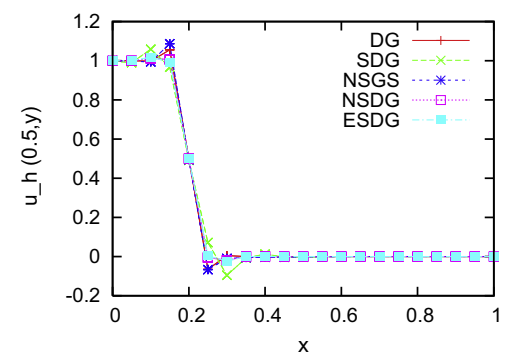

(f) Section at $x=0.5$.

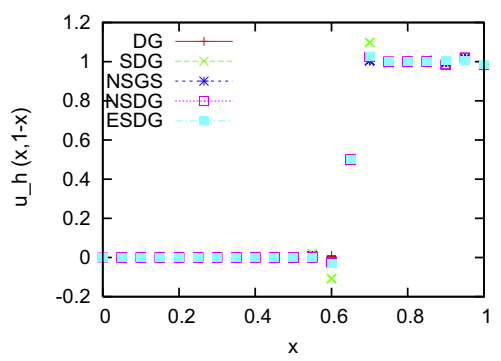

(h) Section at $y=1-x$

Fig. 2. Example 1 : $\varepsilon=10^{-4}, \boldsymbol{\beta}=(1,1)$ and $\sigma=f=0$. 
The first term on the right hand side of (20) provides an $L^{2}$-control of the jumps of the subgrid streamline derivative between adjacent macro elements. The second one gives an $L^{2}$-control of a new (average) subgrid streamline derivative. These two terms depend on the resolution of the resolved scale solution, so that they adapt the edge stabilization according to the situation. However, since neither of these is able to provide enough stability for a wider range of transport parameters, they are combined by using the coefficients $\gamma_{1}$ and $\gamma_{2}$. These coefficients are applied to adjust the contribution of each of the two terms in (20) regarding the formulation accuracy and convergence properties for a variety of problems. We numerically found that $\gamma_{2}$ must be greater than $\gamma_{1}$. Moreover, comprehensive numerical experiments with $0<\gamma_{2}<1$ and $0<\gamma_{1}<\gamma_{2}$ lead to $\gamma_{1}=0.25$ and $\gamma_{2}=0.75$ as the most appropriate coefficients for the analyzed problems.

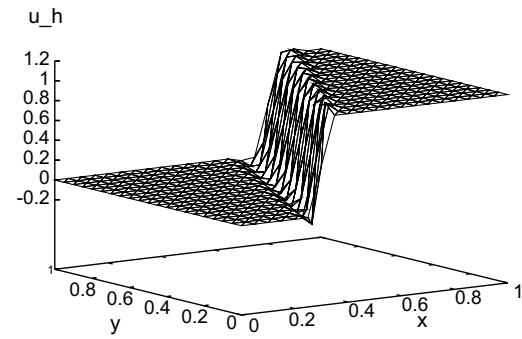

(a) DG solution.

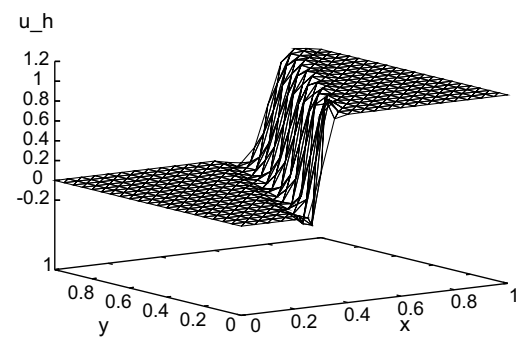

(c) NSDG solution $\left(u_{h}\right)$

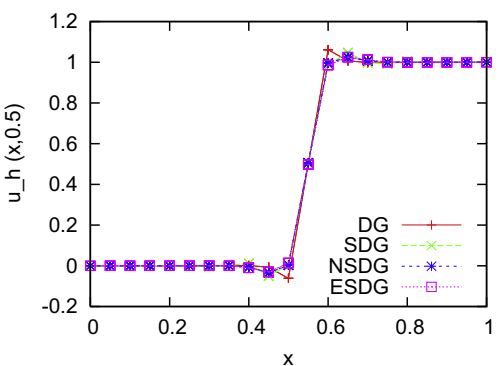

(e) Section at $y=0.5$

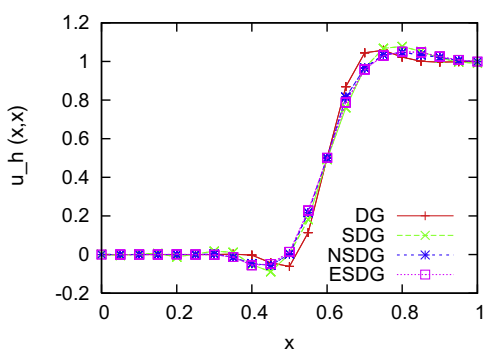

(g) Section at $y=x$
The function $F$ plays the role of adjusting the edge stabilization according to the problem. The non-dimensional parameters $\kappa_{1}=\frac{|\boldsymbol{\beta}|}{\sigma h}$ and $\kappa_{2}=\frac{\varepsilon}{\sigma h^{2}}$ are defined so that they measure the relative importance between the advection and reaction effects and diffusion and reaction effects, respectively. The function $F$ may also depend on the function $\varphi(x)=\max \{0,1-1 / x\}$ and it is defined in the following way:

- advection-diffusion problem: $F=\{|\boldsymbol{\beta}|\}$;

- advection-reaction problem: $F=\left[1-\varphi\left(\kappa_{1}\right)\right] \sigma h+\varphi\left(\kappa_{1}\right)\{|\boldsymbol{\beta}|\}$;

- diffusion-reaction problem: $F=\left[1-\varphi\left(\kappa_{2}\right)\right] \sigma h+\varphi\left(\kappa_{2}\right) \varepsilon / h$.

As far as subgrid formulations are concerned, the interior weighted subgrid penalty-type terms are sufficient to improve stability, as shown in the next section. The nonlinear formulation (19)

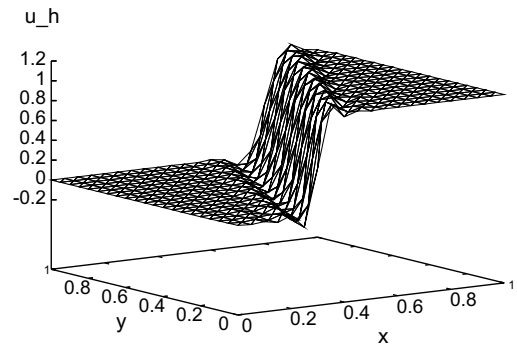

(b) SDG solution $\left(u_{h}\right)$.

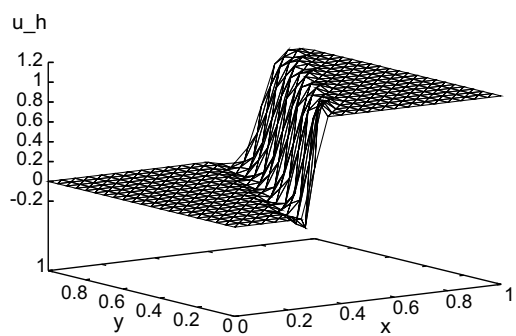

(d) ESDG solution $\left(u_{h}\right)$.

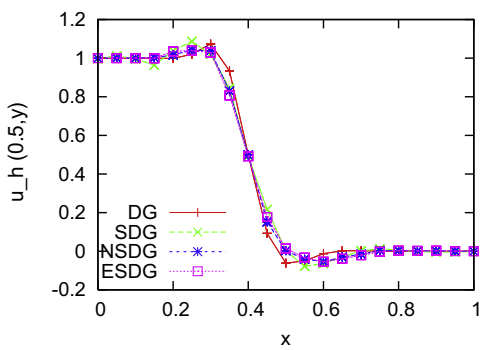

(f) Section at $x=0.5$

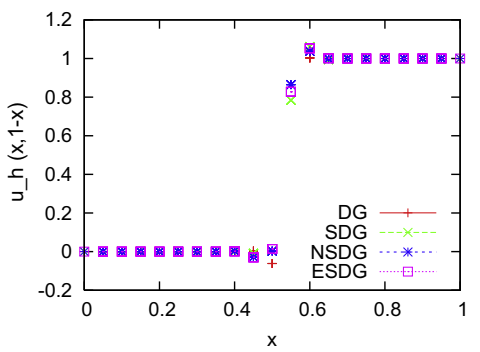

(h) Section at $y=1-x$.

Fig. 3. Example 2 : $\varepsilon=10^{-6}, \boldsymbol{\beta}=(0.5,1)$ and $\sigma=f=0$. 
is solved by using the same iterative procedure introduced in the last section.

\section{Numerical results}

In this section, some classical academic numerical experiments are conducted to illustrate the behavior of the proposed discontinuous formulations applied to advection-diffusion-reaction problems. The new subgrid formulations are compared here with the DG, SDG and NSGS methods. The DG method stands for the formulation developed in [15], in which the broken space is defined on the partition $\mathcal{T}_{h}$. The discontinuous subgrid eddy viscosity method (SDG) was developed in [1] and requires the definition of two parameters, $\varepsilon_{0}$ and $v_{T}$. Here we use $\varepsilon_{0}=1$ and $v_{T}=h_{\min }$, where $h_{\min }$ is the smallest edge length of $T_{h}$. Both DG and SDG are linear methods and present similar computational cost, having the same num-

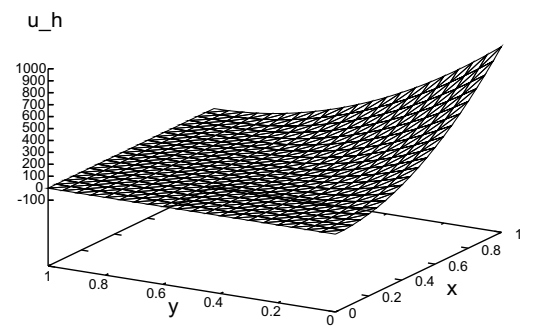

(a) DG solution

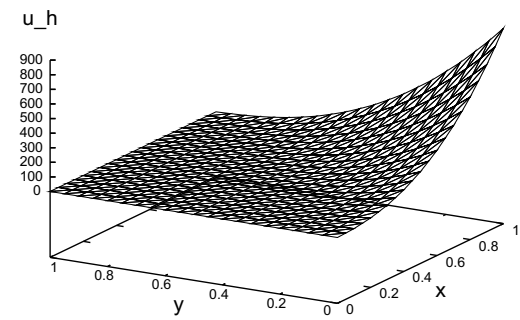

(c) NSDG solution $\left(u_{h}\right)$.

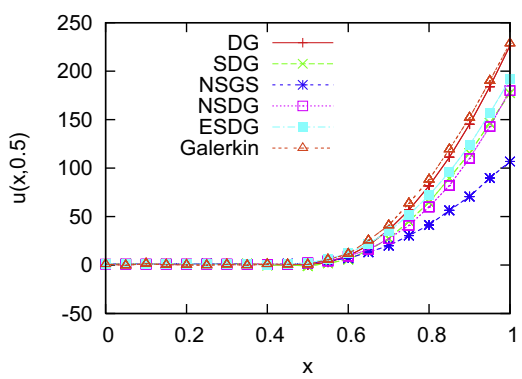

(e) Section at $y=0.5$.

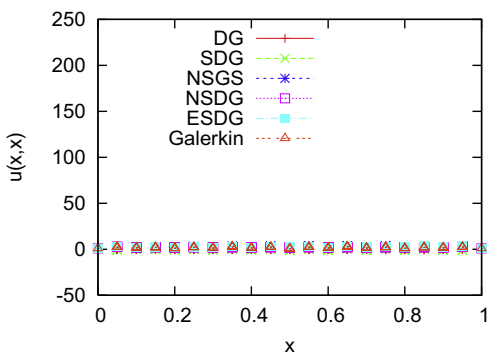

(g) Section at $y=x$ ber of degrees of freedom, about twice that of the proposed formulations. Since a continuous solution is desired for (1), all the approximated solutions are represented in a continuous way, where the solution in each node of the mesh is the average solution of all corresponding degrees of freedom. They are also compared with the solution $u_{h}$ obtained with the NSGS method [25], instead of the resolved scale solution $u_{H}$. The latter is supposed to be free of spurious modes, which are almost completely confined at the subgrid scale. This means that one has to solve a problem two times larger than the resolution of the desired solution. The discontinuous subgrid formulations developed here, as well as the SDG method, do not present the same drawback, so that the following results are presented only for $u_{h}$. In the following computational experiments a regular partition $\mathcal{T}_{h}$ of the domain with 20 divisions in each side is used, unless otherwise indicated. The convergence of the nonlinear methods is attained setting a tolerance equal to $10^{-3}$.

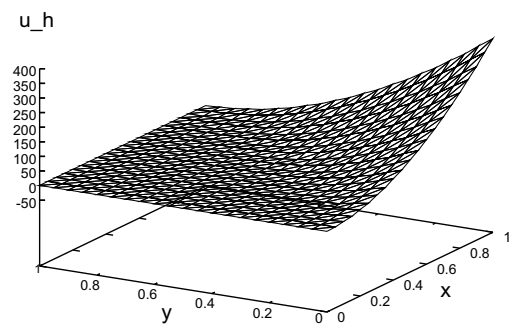

(b) NSGS solution $\left(u_{h}\right)$.

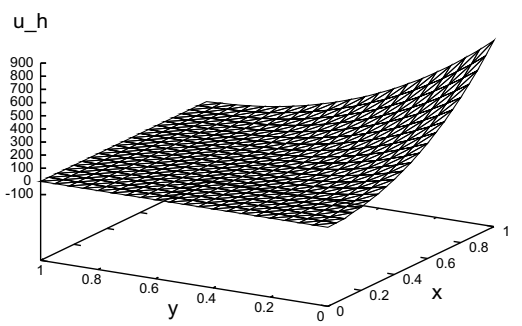

(d) ESDG solution $\left(u_{h}\right)$

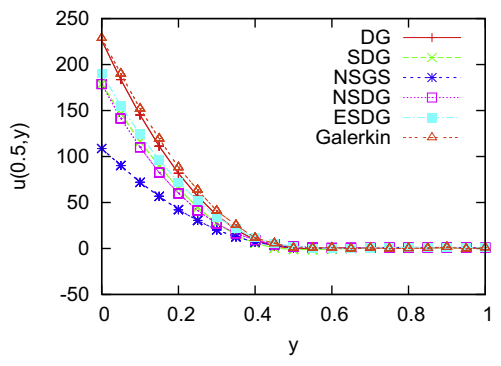

(f) Section at $x=0.5$.

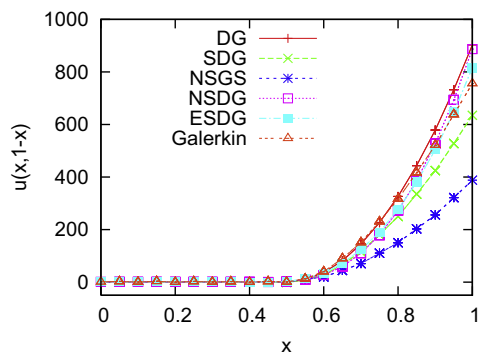

(h) Section at $y=1-x$.

Fig. 4. Example 3. 


\subsection{Advection-diffusion problems}

\subsubsection{Example 1}

This example simulates a two-dimensional advection dominated problem with $\varepsilon=10^{-4}, \beta=(1,1), \sigma=f=0$ in $\Omega=(0,1) \times$ $(0,1)$. The Dirichlet boundary conditions are given by $u(0, y)=$ $u(1, y)=u(x, 1)=0, u(x, 0)=0$ for $x \leqslant 0.3$, and $u(x, 0)=1$ for $x>0.3$. These conditions yield a solution with an interior layer in the direction of the velocity field starting at $(0.3,0)$ and an exponential layer at the outflow, which comprises the side $x=1$ of $\Gamma$.
The Dirichlet boundary conditions are enforced weakly for all the methods in this example. The approximate solutions obtained with the DG, SDG, NSDG and ESDG methods are presented in Fig. 2. The comparison between the NSDG and the ESDG solutions (Fig. 2 (c)-(d)) reveals that they yield similar behavior. For the present case, in which the flow is aligned to the mesh, the DG method is supposed to capture the discontinuities for hyperbolic problems. Since the Peclet number is not very high, Fig. 2(a) shows that the DG mthod gives rise to oscillations in the neighborhood of the internal layer which are damped by using the NSDG and ESDG

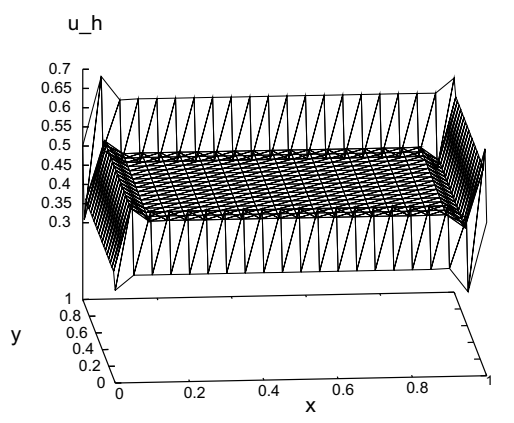

(a) DG solution.

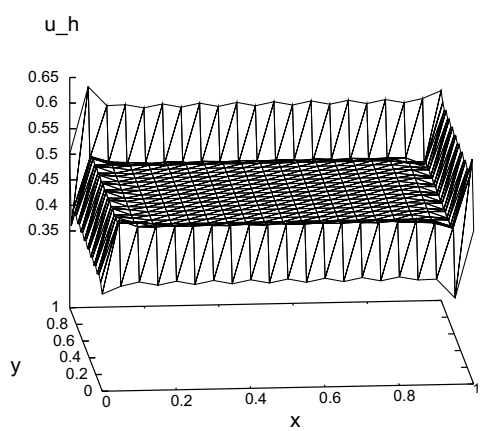

(c) NSDG solution $\left(u_{h}\right)$.

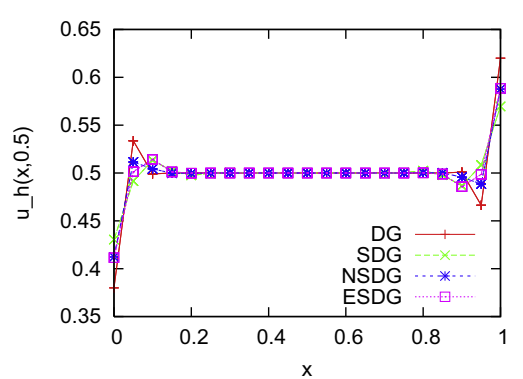

(e) Section at $y=0.5$.

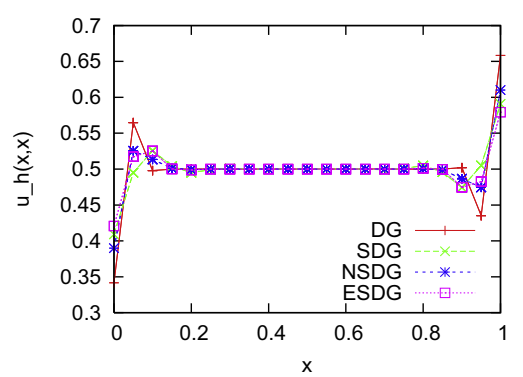

(g) Section at $y=x$.

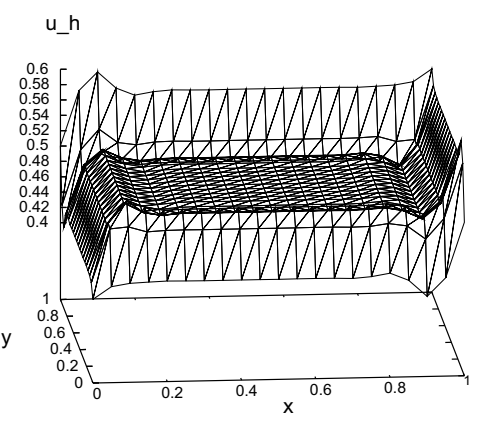

(b) SDG solution $\left(u_{h}\right)$.

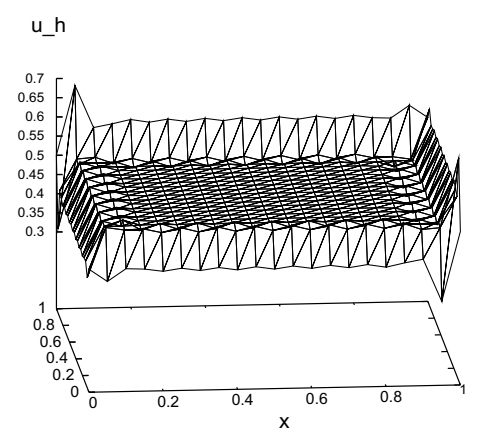

(d) ESDG solution $\left(u_{h}\right)$.

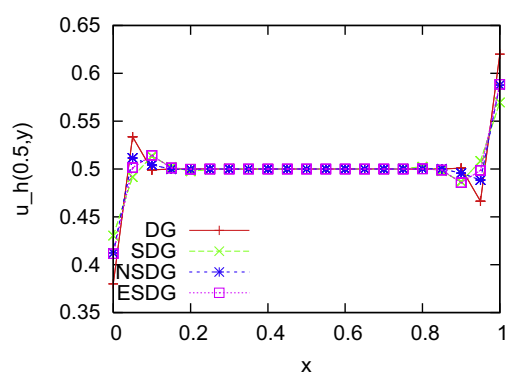

(f) Section at $x=0.5$.

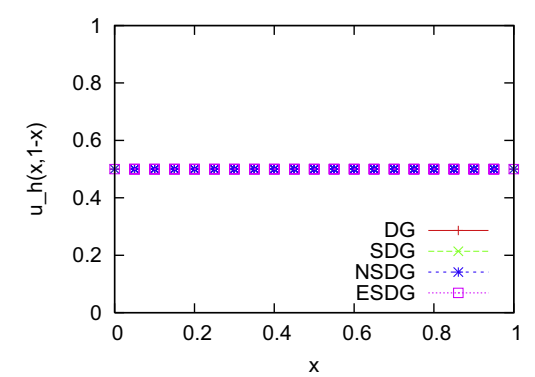

(h) Section at $y=1-x$.

Fig. 5. Example 4 
methods. The worst representation of the internal layer was obtained by using the SDG method. The solution profiles at $y=0.5$, $x=0.5, y=x$ and $y=1-x$, presented in Figs. 2(e)-(h), provide a better comparison among the methods. They also depict the (continuous) NSGS solution for comparison. We may see that NSDG and ESDG solutions are almost free of spurious modes at all sections. Note also that the weakly imposed boundary conditions avoids overshoots in the outflow layer for all methods, although the approximate solutions will satisfy the boundary condition only when the layer is fully resolved.

\subsubsection{Example 2}

This example is similar to Example 1, except that now we consider a smaller diffusivity coefficient $\left(\varepsilon=10^{-6}\right)$ and the velocity field is not aligned with the mesh $(\boldsymbol{\beta}=(0.5,1))$. Fig. 3 shows the approximate solutions obtained using DG, SDG, NSDG and ESDG methods, as well as their profiles at $y=0.5, x=0.5, y=x$ and $y=1-x$. All methods present some oscillations in the neighborhood of the internal layer, which disappear completely only when the mesh is sufficiently fine. However, we may notice some differences: the DG representation of the internal layer is the sharpest one; the behavior of the SDG method improves for the present case (smaller diffusivity coefficient) but it is still worse than that of the DG method; the internal layer is slightly smeared by using either NSDG or ESDG, although the spurious oscillations are smaller for both methods; the behavior of these two methods are much similar.

Table 1

Example 4: convergence behavior.

\begin{tabular}{llllll}
\hline Method & \# dof & \# I & Time (real) & Time (user) & Time (sys) \\
\hline DG & 2400 & 1 & $14^{\prime} 14.330^{\prime \prime}$ & $1^{\prime} 5.544^{\prime \prime}$ & $0^{\prime} 1.688^{\prime \prime}$ \\
SDG & 2400 & 1 & $14^{\prime} 39.448^{\prime \prime}$ & $14^{\prime} 9.945^{\prime \prime}$ & $0^{\prime} 2.108^{\prime \prime}$ \\
NSDG & 1200 & 5 & $3^{\prime} 45.051^{\prime \prime}$ & $3^{\prime} 36.482^{\prime \prime}$ & $0^{\prime} 0.628^{\prime \prime}$ \\
ESDG & 1200 & 8 & $5^{\prime} 38.380^{\prime \prime}$ & $5^{\prime} 30.365^{\prime \prime}$ & $0^{\prime} 0.904^{\prime \prime}$ \\
\hline
\end{tabular}

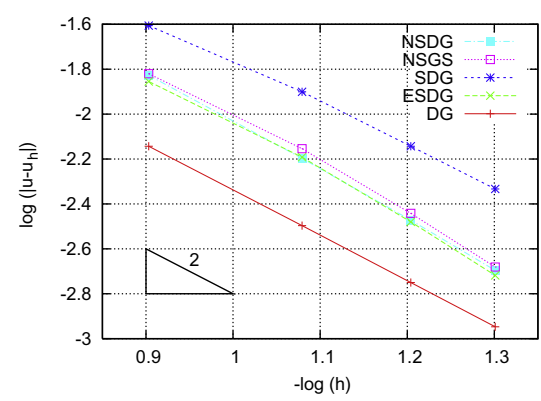

(a) $L^{2}(\Omega)-\varepsilon=10^{-3}$

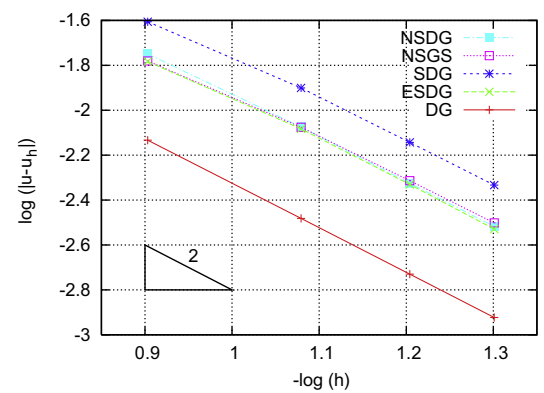

(c) $L^{2}(\Omega)-\varepsilon=10^{-5}$

\subsubsection{Example 3}

This example simulates a two-dimensional advection-diffusion problem with $\varepsilon=10^{-6}, \beta=(1,1), \sigma=f=0$ in $\Omega=(0,1) \times(0,1)$. The Dirichlet boundary conditions are given by $u=1$ on $x=0$ and on $y=1$. The inhomogeneous Neumann boundary conditions are $\varepsilon \frac{\partial u}{\partial y}=1$ on $y=0$ and $\varepsilon \frac{\partial u}{\partial x}=-1$ on $x=1$. Although the solution of this problem presents no layers, it is characterized by an increasing smooth gradient towards the corner $(1,0)$. This problem is suitable for evaluating the behavior of the methods in smooth problems with inhomogeneous Neumann boundary conditions.

The Dirichlet boundary conditions are strongly imposed for all methods in this example. Figs. 4(a)-(d) show the solutions computed with the DG, NSGS, NSDG and ESDG methods, respectively. In the solution profiles (Figs. 4(e)-(h)), the continuous Galerkin solution is included as a reference solution. As expected, both the continuous and discontinuous Galerkin solutions are sharpest since they are less diffusive. The approximate solutions obtained with the NSDG and ESDG methods are very alike and slightly smeared compared to those computed with the Galerkin methods. The ESDG method is slightly less diffusive than the NSDG method. Fig. 4(h) shows that the SDG and the conforming NSGS methods yield smoother approximate solutions, the latter being the least accurate.

\subsection{Reaction-diffusion problem}

\subsubsection{Example 4}

This example simulates a two-dimensional reaction-diffusion problem with $\epsilon=3 \times 10^{-5}, \sigma=1$ and $f=0.5$ in $\Omega=(0,1) \times(0,1)$. The Dirichlet boundary conditions are prescribed on $\Gamma$ as $u(x, 0)=u(0, y)=0$ and $u(x, 1)=u(1, y)=1$. They are enforced weakly for all the methods in this example. Fig. 5 shows the approximated solutions using DG, SDG, NSDG and ESDG methods. The profiles at $y=0.5, x=0.5, y=x$ and $y=1-x$, presented in Figs. 5(e)-(h), show that the DG method gives rise to the biggest oscillations in the neighborhood of the external layers. By using NSDG or ESDG, much smaller oscillations remain so that a better representation of the

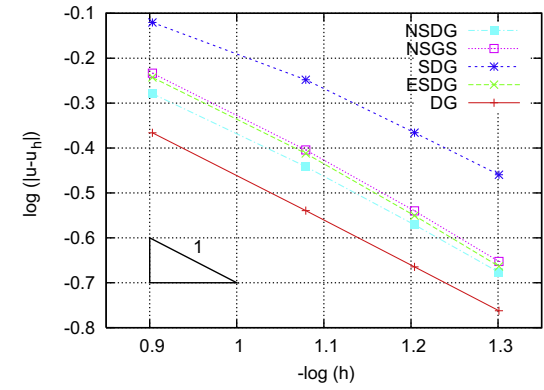

(b) $H^{1}(\Omega)-\varepsilon=10^{-3}$.

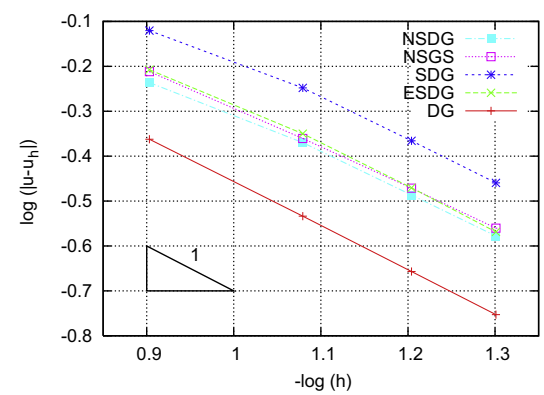

(d) $H^{1}(\Omega)-\varepsilon=10^{-5}$.

Fig. 6. Convergence rates for Example 5. 
solution is obtained. It is worth mentioning that this indicates that the resolved scale solution $u_{H}$ is completely free of spurious modes. One may also notice that the ESDG method is slightly less diffusive.

Now we analyze the role of the nonlinearity associated with the proposed methods. All of them are solved by using a simple direct solver (Gauss). In Table 1 we compare the time necessary to obtain the approximate solution, measured by using the LINUX command time. We also indicate the number of degrees of freedom (\# dof) and the number of iterations (\# I) necessary to converge with respect to the preset tolerance. Table 1 shows that the nonlinear methods are much faster than the linear ones due to the reduction of the total number of degrees of freedom. Also, as the convergence property of the NSDG method is better than that of the ESDG method, the former is the fastest method for this example.

\subsection{Advection-diffusion-reaction problem}

\subsubsection{Example 5}

Finally, in this example we numerically evaluate the convergence properties of the proposed methodologies. It simulates an advection-diffusion-reaction problem with $\beta=(1,0), \sigma=1$, and $\varepsilon=10^{-3}, 10^{-5}$ in $\Omega=(0,1) \times(0,1)$. The source term and the Dirichlet boundary conditions are chosen according to the smooth exact solution, given by

$$
u(x, y)=\exp \left(-\frac{(x-0.5)^{2}}{a}-\frac{3(y-0.5)^{2}}{a}\right), \quad a=0.2 \text {. }
$$

Figs. 6(a)-(d) show the errors of the solutions computed with the methods considered here, measured in the $L^{2}(\Omega)$ and $H^{1}(\Omega)$ norms, respectively, and using meshes with $128,288,512$ and 800 elements. Figs. 6(a)-(b) show the results obtained with $\varepsilon=10^{-3}$, while Figs. 6(c)-(d) refer to $\varepsilon=10^{-5}$. Optimal convergence rates are obtained for all methods, independently of the diffusivity coefficient value. We observe that the DG method is the most accurate for this regular problem, what is expected since the DG method is the least diffusive. The SDG method is the least accurate and both NSDG and NSDG methods have similar behavior.

\section{Conclusion}

Two new discontinuous subgrid scale formulations for the numerical solution of advection-diffusion-reaction problems are developed based on a two-level decomposition of both the approximation spaces and the velocity field. The first subgrid discontinuous formulation proposed here extends the NSGS method $[8,25]$ to discontinuous formulations. The nonlinear subgrid artificial viscosity adjusts the stabilization term that acts only on the subgrid scales and is also used to scale the penalization term associated to the unresolved scale jumps. The edge subgrid formulation provides stabilization by means of a subgrid interior penalty term. In the present approach, a kind of a two-level discontinuous Galerkin formulation is recovered for the resolved scale solution when its gradient vanishes. These methods do not require any user-defined coefficient since the methods themselves adjust their stabilization terms according to the residual of the resolved scale at the element level. The proposed methods have similar accuracy, although the ESDG method is slightly less diffusive than the NSDG method. Their convergence properties are alike and they enjoy the good properties of DG method for handling inhomogeneous boundary conditions for solving advection dominated and reaction dominated transport problems. However, they reduce the number of degrees of freedom since they allow discontinuities only on the resolved scale. Since the resolution of $u_{h}$ is similar to that of $u_{H}$, the decrease in the number of degrees of freedom is highly advantageous as compared with typical DG methods. For mild advection transport problems, the new discontinuous subgrid formulations are more stable since they prevent or reduce spurious oscillations coming from minor scales, which in general are not damped by using the DG method. Optimal convergence rates are obtained using these formulations for regular problems. These features offer an attractive compromise between subgrid scale methods and the classical discontinuous Galerkin method.

\section{Acknowledgments}

This research is partially supported by the Brazilian Government, through the Agency CNPq, Contracts 305730/2007-0 and 306809/2006-0. N.C.B. Arruda acknowledges the Brazilian Agency CAPES (Coordenação de Aperfeiçoamento de Pessoal de Nível Superior) for the graduate scholarship. R.C. Almeida would like to acknowledge the support provided by CAPES, through Grant No. 0858/08-0, and by the J.T. Oden Faculty Fellowship Research Program.

\section{References}

[1] S. Kaya, B. Rivière, A discontinuous subgrid eddy viscosity method for the timedependent Navier-Stokes equations, SIAM J. Numer. Anal. 43 (4) (2005) 15721595 (Electronic).

[2] J.A. Evans, T.J.R. Hughes, G. Sangalli, Enforcement of constraints and maximum principles in the variational multiscale method, Comput. Meth. Appl. Mech. Engrg. 199 (2009) 61-76.

[3] F. Brezzi, L.P. Franca, T.J.R. Hughes, A. Russo, $b=\int g$, Comput. Meth. Appl. Mech. Engrg. 145 (3-4) (1997) 329-339.

[4] T.J.R. Hughes, Multiscale phenomena: Green's functions, the Dirichlet-toNeumann formulation, subgrid scale models, bubbles and the origins of stabilized methods, Comput. Meth. Appl. Mech. Engrg. 127 (1-4) (1995) 387401.

[5] G. Hauke, A. García-Olivares, Variational subgrid scale formulations for the advection-diffusion-reaction equation, Comput. Meth. Appl. Mech. Engrg. 190 (51-52) (2001) 6847-6865.

[6] J.-L. Guermond, Stabilization of Galerkin approximations of transport equations by subgrid modeling, M2AN Math. Model. Numer. Anal. 33 (6) (1999) 1293-1316.

[7] J.-L. Guermond, Subgrid stabilization of Galerkin approximations of linear monotone operators, IMA J. Numer. Anal. 21 (1) (2001) 165-197.

[8] I.P. Santos, R.C. Almeida, A nonlinear subgrid method for advection-diffusion problems, Comput. Meth. Appl. Mech. Engrg. 196 (45-48) (2007) 4771-4778.

[9] E.G. Dutra do Carmo, A.V.C. Duarte, A discontinuous finite element-based domain decomposition method, Comput. Meth. Appl. Mech. Engrg. 190 (8-10) (2000) 825-843.

[10] E.G. Dutra do Carmo, A.V.C. Duarte, New formulations and numerical analysis of discontinuous Galerkin methods, Comput. Appl. Math. 21 (3) (2002) 661715.

[11] H. Luo, J.D. Baum, R. Löhner, A discontinuous Galerkin method based on a taylor basis for the compressible flows on arbitrary grids, J. Comput. Phys. 227 (2008) 8875-8893.

[12] R. Biswas, K.D. Devine, J.E. Flaherty, Parallel, adaptive finite element methods for conservation laws, Appl. Numer. Math. 14 (1994) 255-283.

[13] H. Hoteit, P. Ackerer, R. Mosé, J. Erhel, B. Philippe, New two-dimensional slope limiters for discontinuous Galerkin methods on arbitrary meshes, Int. J. Numer. Meth. Engng. 61 (2004) 2566-2593.

[14] W. Klieber, B. Rivière, Adaptive simulations of two-phase flow by discontinuous Galerkin methods, Comput. Meth. Appl. Mech. Engrg. 196 (2006) 404-419.

[15] P. Houston, C. Schwab, E. Süli, Stabilized hp-finite element methods for firstorder hyperbolic problems, SIAM J. Numer. Anal. 37 (5) (2000) 1618-1643.

[16] F. Brezzi, B. Cockburn, L.D. Marini, E. Süli, Stabilization mechanisms in discontinuous Galerkin finite element methods, Comput. Meth. Appl. Mech. Engrg. 195 (25-28) (2006) 3293-3310.

[17] P.F. Antonietti, F. Brezzi, L.D. Marini, Bubble stabilization of discontinuous Galerkin methods, Comput. Meth. Appl. Mech. Engrg. 198 (21-26) (2009) 1651-1659. Advances in Simulation-Based Engineering Sciences - Honoring J. Tinsley Oden.

[18] F.A. Rochinha, G.B. Alvarez, E.G.D. do Carmo, A.F.D. Loula, A locally discontinuous enriched finite element formulation for acoustics, Commun. Numer. Meth. Engng. 23 (2007) 623-637.

[19] E. Burman, A unified analysis for conforming and nonconforming stabilized finite element methods using interior penalty, SIAM J. Numer. Anal. 43 (5) (2005) 2012-2033.

[20] I. Babuška, M. Zlámal, Nonconforming elements in finite element with penalty, SIAM J. Numer. Anal. 10 (1973) 863-875.

[21] J. Douglas Jr., T. Dupont, Interior penalty procedures for elliptic and parabolic Galerkin methods, in: Computing Methods in Applied Sciences (Second 
International Symposium, Versailles, 1975), Lecture Notes in Physics, vol. 58 , Springer, Berlin, pp. 207-216.

[22] E. Burman, P. Hansbo, Edge stabilization for Galerkin approximations of convection-diffusion-reaction problems, Comput. Meth. Appl. Mech. Engrg. 193 (15-16) (2004) 1437-1453.

[23] E. Burman, P. Hansbo, Edge stabilization for the generalized Stokes problem: a continuous interior penalty method, Comput. Meth. Appl. Mech. Engrg. 195 $(19-22)$ (2006) 2393-2410.
[24] J.-L. Guermond, J.T. Oden, S. Prudhomme, Mathematical perspectives on large eddy simulation models for turbulent flows, J. Math. Fluid Mech. 6 (2004) 194248.

[25] I.P. Santos, R.C. Almeida, A free parameter subgrid scale model for transport problems, Comput. Meth. Appl. Mech. Engrg. submitted for publication.

[26] A. Ern, J.-L. Guermond, Theory and practice of finite elements, Applied Mathematical Science, vol. 159, Springer-Verlag, 2004. 